M. Nakai

Nagoya Math. J.

Vol. 50 (1973), 67-87

\title{
ORDER COMPARISONS ON CANONICAL ISOMORPHISMS
}

\author{
MITSURU NAKAI
}

Consider a nonnegative Hölder continuous 2-form $P(z) d x d y(z=x+i y)$ on a connected Riemann surface $R$. We denote by $P(R)$ the linear space of solutions $u$ of the equation $\Delta u=P u$ on $R$ and by $P X(R)$ the subspace of $P(R)$ consisting of those $u$ with a certain boundedness property $X$. We also use the standard notations $H(R)$ and $H X(R)$ for $P(R)$ and $P X(R)$ with $P \equiv 0$. As for $X$ we take $B$ to mean the finiteness of the supremum norm $\|u\|=\sup _{R}|u|, D$ the finiteness of the Dirichlet integral $D(u)=$ $\int_{R} d u \wedge^{*} d u, E$ the finiteness of the energy integral $E(u)=\int_{R}\left(d u \wedge^{*} d u\right.$ $\left.+u^{2}(z) P(z) d x d y\right)$, and their nontrivial combinations $B D$ and $B E$. Let $Q(z) d x d y$ be another 2 -form of the same kind. We say that $P X(R)$ is canonically isomorphic to $Q X(R)$ if there exists a linear isomorphism $T$ of $P X(R)$ onto $Q X(R)$ such that $u$ and $T u$ have the same ideal boundary values for every $u$ in $P X(R)$ in the sense that $|u-T u|$ is dominated by a potential on $R$, i.e. a nonnegative superharmonic function whose greatest harmonic minorant is zero. In the pioneering work [14] concerning canonical isomorphisms, Royden proved the following order comparison theorem: If there exists a constant $c \geq 1$ such that

$$
c^{-1} P(z) \leq Q(z) \leq c P(z)
$$

on hyperbolic $R$ except possibly for a compact subset $K$ of $R$, then $P B(R)$ and $Q B(R)$ are canonically isomorphic. In this connection we wish to discuss the following two questions:

$1^{\circ}$. Is the condition (1) also sufficient for $P X(R)$ and $Q X(R)$ to be canonically isomorphic for $X=D, E, B D$, and $B E$ ?

$2^{\circ}$. In the affirmative case how large can we make the exceptional set $K$ in (1) for $X=B, D, E, B D$, and $B E$ ?

Received September 12, 1972. 
We shall actually show that the answer to $1^{\circ}$ is affirmative. To give the complete answer to $2^{\circ}$ is probably very difficult. In this paper we shall considerably enlarge the class of exceptional sets as follows. Let $W$ be an open subset of $R$ with an analytic relative boundary $\partial W$ and consider the relative class $H X(W ; \partial W)=\{u \in H X(W) \cap C(R) ; u \mid R-W=0\}$ for $X=B, D$, and $B D$. The Heins injection $\lambda_{H}: H X(W ; \partial W) \rightarrow H X(R)$ is given by $\lambda_{H} u=\lim _{\Omega \rightarrow R} H_{u}^{\Omega}$. We say that a subset $K$ of $R$ is $X$-negligible $(X=B, D$, and $B D$ ) if either $R$ is parabolic or there exists a $W$ with $K \subset R-W$ such that $\lambda_{H}: H X(W ; \partial W) \rightarrow H X(R)$ is surjective. We will see that a subset $K$ of hyperbolic $R$ is $B$-negligible ( $B D$-negligible, resp.) if and only if there exists a potential (Dirichlet finite potential, resp.) $p$ such that $p \geq 1$ on $K$. Such a simple characterization for $D$-negligible sets is not available. We only know that if there exists a Dirichlet finite potential $p$ harmonic outside a compact set of $R$ such that $p \geq 1$ on $K$, then $K$ is $D$-negligible. By these criterions we see that compact sets are trivial examples of our negligible sets. The purpose of this paper is to contribute to the solution of questions $1^{\circ}$ and $2^{\circ}$ as follows:

ORDER COMPARISON THEOREM. Let $R$ be a hyperbolic connected Riemann surface. If (1) is valid on $R$ except possibly for a B-negligible subset of $R$, then $P B(R)$ and $Q B(R)$ are canonically isomorphic; if (1) is valid on $R$ except possibly for a $B D$-negligible subset of $R$, then $P B D(R)$ $(P B E(R)$, resp.) and $Q B D(R)(Q B E(R)$, resp.) are canonically isomorphic; if (1) is valid on $R$ except possibly for a $D$-negligible subset of $R$, then $P D(R)(P E(R)$, resp. $)$ and $Q D(R)(Q E(R)$, resp.) are canonically isomorphic.

We excluded parabolic $R$ since in such a case $P X(R)=\{0\}$ for every $X=B, D, E, B D, B E$ and $P \neq \equiv$, and $H X(R)=$ constants $\}$. In nos. 1-4, we shall prove that the theorem is true if the exceptional set in (1) is empty. We consider canonical injections: $P X(R) \rightarrow Q X(R)$ and reduction operators: $P X(R) \rightarrow H X(R)$ as preparations for considering canonical isomorphisms. The notion of quasipotential will be introduced and prove to be useful; at least it is a convenient terminolgy. In nos. 5-7, the surjectiveness of canonical extensions $P X(W ; \partial W) \rightarrow P X(R)$ will be discussed. After these preparations, the proof of our order comparison theorem will be given in no. 8. Although our main concern in this paper is the order comparisons, we will append a sketch of the other kind of 
important criterion, the integral comparisons, for the existence of canonical isomorphisms in nos. 9-10. The first integral comparison theorem in no. 9 generalizes those thus far known. The second one in no. 10 completely characterizes the surjectiveness of reduction operators: $P X(R)$ $\rightarrow H X(R)$ for $X=B, B D$, and $B E$. Methodologically the use of the compactification theory of Riemann surfaces would give more clearer geometric intuitive insight to the whole discussion in this paper. However to make the description as elementary as possible we will intentionally avoid its use even if it is preferable.

\section{Canonical Injections}

1. It will be convenient to include disconnected but separable surfaces in our considerations. Therefore we assume that our Riemann surface $R$ is either connected or an open subset of a connected Riemann surface. By a regular open set we mean a finite union of closure disjoint regular regions in $R$. We use the notation $\Omega$ for regular open sets. The totality $\{\Omega\}$ of regular open sets $\Omega$ of $R$ forms a directed net by inclusions converging to $R$. A 2-form $P(z) d x d y(z=x+i y)$ on $R$ is said to be Hölder continuous if, for each parametric disk $(U, z)$, there exist constant $K=K(U, z) \in(0, \infty)$ and $\alpha=\alpha(U, z) \in(0,1]$ such that $\left|P\left(z_{1}\right)-P\left(z_{2}\right)\right|$ $\leq K\left|z_{1}-z_{2}\right|^{\alpha}$ for every pair of points $z_{1}$ and $z_{2}$ in $U$. We say that $P(z) d x d y$ is nonnegative, $P(z) d x d y \geq 0$ or $P(z) \geq 0$ in notation, if, for each parametric disk $(U, z), P(z) \geq 0$ for every $z$ in $U$. These are well defined since such properties are invariant under the change of local parameters. In particular the order $P(z) d x d y \geq Q(z) d x d y$ or $P(z) \geq Q(z)$ can be defined between two 2-forms by $(P(z)-Q(z)) d x d y \geq 0$. The 2-form $P(z) d x d y$ is (identically) zero if, for each parametric disk $(U, z), P(z) \equiv 0$ in $U$. We simply denote this by $P \equiv 0$. Nonnegative Hölder continuous 2-forms on $R$ will be denoted by $P(z) d x d y, Q(z) d x d y$, etc.

We denote by $P_{\varphi}^{\Omega}$ the solution of the Dirichlet problem of the equation $\Delta u=P u$ on $\Omega$ with a continuous boundary values $\varphi$ on the relative boundary $\partial \Omega$, i.e. $P_{\varphi}^{\Omega} \in P(\Omega) \cap C(\bar{\Omega})$ with $P_{\varphi}^{\Omega} \mid \partial \Omega=\varphi$, where $P(\Omega)$ is the linear space of $C^{2}$ solutions of $\Delta u=P u$ on $\Omega$. By the limiting process we can define $P_{\varphi}^{\Omega}$ even for upper- or lower- semicontinuous functions $\varphi$. We also use the standard notation $H_{\varphi}^{\Omega}$ and $H(\Omega)$ for $P_{\varphi}^{\Omega}$ and $P(\Omega)$ with $P \equiv 0$. Let $G_{\Omega}(z, \zeta)$ be the harmonic Green's function on $\Omega$. If $\Omega$ is connected, then there is no question about its definition. If $\Omega=\bigcup_{i=1}^{n} \Omega_{i}$ 
with $\Omega_{i}$ connected and $\bar{\Omega}_{i} \cap \bar{\Omega}_{j}=\phi(i \neq j)$, then $G_{\Omega}(z, \zeta)=G_{\Omega_{i}}(z, \zeta)$ for $z, \zeta \in \Omega_{i}$ and $G_{\Omega}(z, \zeta)=0$ for $z \in \Omega_{i}$ and $\zeta \in \Omega_{j}(i \neq j)$. Then

$$
P_{\varphi}^{\Omega}=H_{\varphi}^{\Omega}-\frac{1}{2 \pi} \int_{\Omega} G(\cdot, \zeta) P_{\varphi}^{\Omega}(\zeta) P(\zeta) d \xi d \eta,
$$

where $\zeta=\xi+i \eta$, and the Dirichlet integral $D_{\Omega}\left(P_{\varphi}^{\Omega}\right)=\int_{\Omega} d P_{\varphi}^{\Omega} \wedge * d P_{\varphi}^{\Omega}$ is given by

$$
D_{\Omega}\left(P_{\varphi}^{\Omega}\right)=D_{\Omega}\left(H_{\varphi}^{\Omega}\right)+\frac{1}{2 \pi} \int_{\Omega \times \Omega} G_{\Omega}(z, \zeta) P_{\varphi}^{\Omega}(z) P_{\varphi}^{\Omega}(\zeta) P(z) d x d y P(\zeta) d \xi d \eta
$$

(cf. e.g. $[9,10])$. Since the energy integral $E_{\Omega}(u)=E_{\Omega}^{P}(u)=D_{\Omega}(u)+$ $\int_{\Omega} u^{2}(z) P(z) d x d y$ is the variation whose Euler-Lagrange equation is $\Delta u=P u$, we have the so-called energy principle (Dirichlet principle):

$$
E_{\Omega}\left(P_{\varphi}^{\Omega}\right)=\min \left(E_{\Omega}(u) ; u \in C(\bar{\Omega}) \cap C^{w}(\Omega), u \mid \partial \Omega=\varphi\right),
$$

where $C^{w}$ is the class of weakly differentiable functions (cf. e.g. [1]). Another simple but important fact which will be used repeatedly is that $|u|$ and $u \cup 0=\max (u, 0)$ are subharmonic for every $u \in P(R)$. Since $\Delta u=P u \geq 0$ in $\{u>0\}, u \cup 0=u$ is subharmonic in $\{u>0\}$, and so is $u \cup 0=0$ in $\{u<0\}$. The submean value property is clearly valid at each point of $\{u=0\}$ for $u \cup 0$. Therefore $u \cup 0$ and $(-u) \cup 0$ are subharmonic on $R$, and so is $|u|=u \cup 0+(-u) \cup 0$. A potential $p$ on $R$ is a nonnegative superharmonic function whose greatest harmonic minorant is zero. If $\Omega \subset \Omega^{\prime}$, then $0 \leq H_{p}^{\Omega^{\prime}} \leq H_{p}^{\Omega}$ and $\lim _{\Omega \rightarrow R} H_{p}^{\Omega}$ is a nonnegative harmonic function on $R$ dominated by $p$. Thus $\lim _{\Omega \rightarrow R} H_{p}^{\Omega}=0$ and actually this is the defining property for a nonnegative superharmonic function $p$ to be a potential. A function $f$ on $R$ will be referred to as a quasipotential if $|f|$ is majorated by a potential $p=p_{f}$. Clearly the class of quasipotentials (potentials, resp.) forms a linear (additive, resp.) space. Since $\left|P_{f}^{\Omega}\right| \leq P_{|f|}^{\Omega} \leq P_{p}^{\Omega} \leq H_{p}^{\Omega}$ with $p=p_{f}$, we have

$$
\lim _{\Omega \rightarrow R} P_{f}^{\Omega}=\lim _{\Omega \rightarrow R} P_{|f|}^{\Omega}=0
$$

for every upper- or lower- semicontinuous quasipotential $f$ on $R$. The following fact will also be used repeatedly: If $f$ is a quasipotential such that $|f|$ is subharmonic, then $f=0$. This follows from (5) and the inequality $|f| \leq H_{|f|}^{\Omega}$. 
We denote by $P B(R)$ the subspace of $P(R)$ consisting of solutions $u$ with the finite supremum norms: $\|u\|=\|u\|_{R}=\sup _{R}|u|<\infty$. The notation $P D(R)$ is used for the subspace consisting of solutions $u$ with the finite Dirichlet integrals $D(u)=D_{R}(u)<\infty$. The subspace $P E(R)$ consists of solutions $u$ such that the energy integrals $E(u)=E_{R}(u)=E_{R}^{P}(u)<\infty$. Similarly $P B D(R)=P B(R) \cap P D(R)$ and $P B E(R)=P B(R) \cap P E(R)$. Contrary to $P B(R)$ and $P D(R)$, the scale $E$ for which every solution in $P E(R)$ is finite varys according to $P$. We use the standard notations $H X(R)$ for $P X(R)$ with $P \equiv 0(X=B, D, E, B D, B E)$. In this case $E(u)=D(u)$ and thus e.g. $H E(R)=H D(R)$. We denote by $P X(R)^{+}$the subset of $P X(R)$ consisting of nonnegative solutions. It is of fundamental importance that $P X(R)^{+}$generates $P X(R)$ for $X=B, D, E, B D$, and $B E$ (cf. e.g. [14], [7], [2]). We say that $P X(R)$ and $Q X(R)(X=B, D, E, B D, B E)$ are canonically isomorphic if there exists a linear isomorphism $T$ of $P X(R)$ onto $Q X(R)$ such that $u-T u$ is a quasipotential for every $u \in P X(R)$. The operator $T$ is unique, order preserving, and isometric, and will be referred to as the canonical isomorphism. In fact, if $T^{\prime}$ is another such operator, then $\left|T u-T^{\prime} u\right| \leq|u-T u|+\left|u-T^{\prime} u\right|$ and thus $\left|T u-T^{\prime} u\right|$ is a quasipotential. Since $\left|T u-T^{\prime} u\right|$ is subharmonic, $T u=T^{\prime} u$ for every $u \in P X(R)$. Suppose $u \in P X(R)^{+}$. Since $T u \geq u-|u-T u|, Q_{u}^{a} \geq 0$, and $Q_{T u}^{\Omega}=T u$, we have $T u \geq Q_{u}^{\Omega}-Q_{|u-T u|}^{\Omega} \geq-Q_{|u-T u|}^{\Omega}$ on $\Omega$. On letting $\Omega \rightarrow R$, we conclude by (5) that $T u \geq 0$, i.e. $T u \in Q X(R)^{+}$. For general $u \in P X(R)$, observe that $|T u| \leq|u|+|u-T u|$. Since $H_{|u|}^{a} \leq\|u\|_{\partial \Omega} \leq\|u\|$, by the maximum principle for subharmonic functions we see that $|T u| \leq H_{|T u|}^{\Omega} \leq$ $\|u\|+H_{|u-T u|}^{\Omega}$ on $\Omega$. Again by (5) we conclude that $\|T u\| \leq\|u\|$. Similarly $|u| \leq|T u|+|u-T u|$ implies $|u| \leq H_{|u|}^{\Omega} \leq\|T u\|+H_{|u-T u|}^{\Omega}$ and then $\|u\| \leq\|T u\|$. Therefore $\|T u\|=\|u\| \leq \infty$.

2. To study the existence of canonical isomorphism, it is convenient to consider a canonical injection $T=T_{Q, P}$ of $P X(R)$ into $Q X(R)(X=$ $B, D, E, B D, B E)$. It is a linear operator from $P X(R)$ into $Q X(R)$ such that $u-T u$ is quasipotential for every $u \in P X(R)$. It is actually injective. If $T u=T v$, then $|u-v| \leq|u-T u|+|v-T v|$ shows that the subharmonic function $|u-v|$ is a quasipotential and hence $u=v$. By exactly the same proof as in the last part of no. 2 we see that the canonical injection is unique, order preserving, and isometric. If $T_{P_{2}, P_{1}}$ and $T_{P_{3}, P_{2}}$ exist for $X$, then $T_{P_{3}, P_{1}}$ exists for $X$ and 


$$
T_{P_{3}, P_{1}}=T_{P_{3}, P_{2}} \circ T_{P_{2}, P_{1}} \text {. }
$$

In fact, $T=T_{P_{3}, P_{2}} \circ T_{P_{2}, P_{1}}$ is a linear operator from $P_{1} X(R)$ into $P_{3} X(R)$.

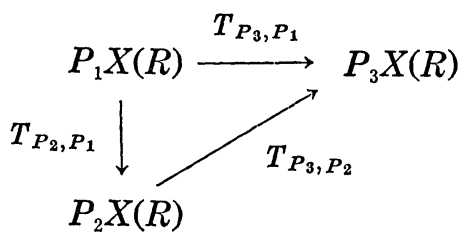

Let $u \in P_{1} X(R)$ and set $v=T_{P_{2}, P_{1}} u \in P_{2} X(R)$. Since $|u-T u| \leq\left|u-T_{P_{2}, P_{1}} u\right|$ $+\left|v-T_{P_{3}, P_{2}} v\right|, u-T u$ is a quasipotential and thus $T$ is the canonical injection $T_{P_{3}, P_{1}}$. To determine pairs $(P, Q)$ such that $T_{Q, P}$ exists is very important but a difficult problem. For our present purpose we will only prove that pairs $(P, Q)$ with $P \geq Q$ have this property, which is originally obtained by Royden [14] and in abstract setting by Loeb [4] for $X=B$ (cf. also Maeda [5], Glasner-Katz [2]). It is convenient to discuss first the existence of $T_{P}=T_{0, P}$, which is in particular referred to as the reduction operator. The term is employed by Singer [16] to suggest that the operator $T_{P}$ reduces the study of the class $P X(R)$ to that of more manageable class $H X(R)$. In this context, it is also important to determine $P$ such that $T_{P}$ is surjective, i.e. $P X(R)$ is canonically isomorphic to $H X(R)$. For $X=B, B D$, and $B E$, a complete answer is known (cf. Appendix, no. 10). For $X=D$ and $E$, we only have partial informations (cf. Singer [17], [11]). Here we only prove that the reduction operator $T_{P}$ always exists uniquely for every $P$. Let $u \in P X(R)$. If $X=D$ or $E$, then $D(u)<\infty$ and the harmonic decomposition of RoydenBrelot (cf. e.g. [1], [15]) assures that $\lim _{\Omega \rightarrow R} H_{u}^{\Omega} \in H D(R)$. If $X=B$, then let $u=u_{1}-u_{2}$ with $u_{i} \in P B(R)^{+}(i=1,2)$. Since $\left\{H_{u_{j}}^{\Omega}\right\}$ is increasing, $\left|H_{u_{j}}^{\Omega}\right| \leq\left\|u_{i}\right\|$, and $H_{u}^{\Omega}=H_{u_{1}}^{\Omega}-H_{u_{2}}^{\Omega}$, we also conclude that $\lim _{\Omega \rightarrow R} H_{u}^{\Omega} \in H B(R)$. Therefore the linear operator $T_{P}$ of $P X(R)$ into $H X(R)$ can be defined by

$$
T_{P} u=\lim _{\Omega \rightarrow R} H_{u}^{\Omega}
$$

for every $u \in P X(R)(X=B, D, E, B D, B E)$. To see that (7) is actually the reduction operator, let $u=u_{1}-u_{2}$ with $u_{i} \in P X(R)^{+}(i=1,2)$. Let $h_{i}$ be an arbitrary harmonic function with $h_{i} \geq u_{i}$. By the subharmonicity of $u_{i}, u_{i} \leq H_{u_{i}}^{\Omega} \leq h_{i}$ and thus $T_{P} u_{i} \leq h_{i}$. This means that $T_{P} u_{i}$ is the least harmonic majorant of $u_{i}$. Therefore $T_{P} u_{i}-u_{i}$ is a potential $(i=1,2)$ and $\left|u-T_{P} u\right| \leq\left|u_{1}-T_{P} u_{1}\right|+\left|u_{2}-T_{P} u_{2}\right|$ and a fortiori $u-T_{P} u$ is a quasipotential. 
Besides (7) the following representation of $T_{P}$ is also useful. We define the harmonic Green's function $G_{R}(z, \zeta)$ on a Riemann surface $R$ as follows. Let $R=\cup_{n} R_{n}$ be the decomposition into connected components $R_{n}$. Thus $R_{n}$ is a connected Riemann surface. For $z, \zeta \in R_{n}$, let $G_{R}(z, \zeta)=G_{R_{n}}(z, \zeta)$ the usual harmonic Green's function on $R_{n}$ if $R_{n}$ is hyperbolic and $G_{R}(z, \zeta)=+\infty$ if $R_{n}$ is parabolic. For $z \in G_{n}$ and $\zeta \in R_{m}$ $(n \neq m)$, we set $G_{R}(z, \zeta)=0$. Therefore

$$
G_{R}(z, \zeta)=\lim _{\Omega \rightarrow R} G_{\Omega}(z, \zeta)
$$

Let $u \in P X(R)$. Then

$$
T_{P} u=u+\frac{1}{2 \pi} \int_{R} G_{R}(\cdot, \zeta) u(\zeta) P(\zeta) d \xi d \eta .
$$

More precisely if $P \not \equiv 0$ on $R_{n}$ and $P X\left(R_{n}\right)$ contains a nonzero function then $G_{R}(z, \zeta) \not \equiv \infty$ for $z$ and $\zeta$ in $R_{n}$. Since $P_{u}^{\Omega}=u$, we see by (2) that

$$
H_{u}^{\Omega}=u+\frac{1}{2 \pi} \int_{\Omega} G_{\Omega}(\cdot, \zeta) u(\zeta) P(\zeta) d \xi d \eta
$$

If $u \in P X(R)^{+}$, then the integrand is increasing with respect to $\Omega$ and therefore the Lebesgue-Fatou theorem implies that

$$
T_{P} u=u+\frac{1}{2 \pi} \int_{R}\left(\lim _{\Omega \rightarrow R} G^{\Omega}(\cdot, \zeta)\right) u(\zeta) P(\zeta) d \xi d \eta
$$

Since $P X(R)^{+}$generates $P X(R)$, we see the validity of (8). By (3) we also have

$$
D(u)=D\left(T_{P} u\right)+\frac{1}{2 \pi} \int_{R \times R} G_{R}(z, \zeta) u(z) u(\zeta) P(z) d x d y P(\zeta) d \xi d \eta
$$

for $u \in P X(R)$ such that the integral has definite meaning. This is the case for $u \in P X(R)^{+}$for every $X$ and for $u \in P X(R)(X=D, E)$; and if $u \in P X(R)(X=D, E)$, then each term in (9) is finite. By the energy principle (Dirichlet principle)

$$
\langle u, u\rangle_{R}^{P}=\int_{R \times R} G_{R}(z, \zeta) u(z) u(\zeta) P(z) d x d y P(\zeta) d \xi d \eta \geq 0
$$

as soon as the integral is definite. The quantity is referred to as the $P-G r e e n$ energy and the relation (10) is known as that the Green kernel is of positive type in the Green potential theory. 
If the canonical injection $T_{Q, P}$ of $P X(R)$ into $Q X(R)$ exists, then, since $\left|T_{Q, P} u-u\right|$ and $\left|T_{Q}\left(T_{Q, P} u\right)-T_{Q, P} u\right|$ are quasipotentials, the inequality $\left|T_{Q}\left(T_{Q, P} u\right)-u\right| \leq\left|T_{Q}\left(T_{Q, P} u\right)-T_{Q, P} u\right|+\left|T_{Q, P} u-u\right|$ shows that $u-T_{Q}\left(T_{Q, P} u\right)$ is a quasipotential. Therefore, by the uniqueness of the reduction operator,

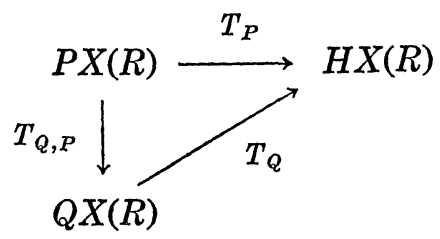

the linear operator $T_{Q} \circ T_{Q, P}$ from $P X(R)$ into $H X(R)$ must be the reduction operator $T_{P}$, i.e.

$$
T_{P}=T_{Q} \circ T_{Q, P} .
$$

We also have an analogue of (7): If $T_{Q, P}$ for $X$ exists, then

$$
T_{Q, P} u=\lim _{\Omega \rightarrow R} Q_{u}^{\Omega}
$$

for every $u \in P X(R)$. In fact, $Q_{u}^{\Omega}=Q_{T_{Q}, P u}^{\Omega}+Q_{u-T_{Q, P u}}^{\Omega}$ and (5) imply the relation. From (11) it follows that $T_{Q, P}$ from $P X(R)$ into $Q X(R)$ exists if and only if

$$
T_{P}(P X(R)) \subset T_{Q}(Q X(R)),
$$

and in this case $T_{Q, P}=T_{Q}^{-1} \circ T_{P}$. The necessity is clear by (11). Conversely, if (13) is valid, then the inverse $T_{Q}^{-1}$ of $T_{Q}$ from $T_{Q}(Q X(R))$ onto $Q X(R)$ can be defined on $T_{P}(P X(R))$. Let $u \in P X(R)$ and set $v=T_{P} u$ and $w=T_{Q}^{-1} v \in Q X(R)$. Then $\left|u-T_{Q}^{-1}\left(T_{P} u\right)\right|=|u-w| \leq|w-v|+|u-v|=$ $\left|w-T_{Q} w\right|+\left|u-T_{P} u\right|$ shows that $u-T_{Q}^{-1}\left(T_{P} u\right)$ is a quasipotential for every $u \in P X(R)$. Therefore the operator $T_{Q}^{-1} \circ T_{P}$ is the canonical injection from $P X(R)$ into $Q X(R)$. From this we see that if the canonical injections $T_{Q, P}$ and $T_{P, Q}$ exist for $X$, then they are canonical isomorphisms, i.e. $T_{P, Q}=T_{Q, P}^{-1}$ and $T_{Q, P}=T_{P, Q}^{-1}$. In this case, we see by (13)

$$
T_{P}(P X(R))=T_{Q}(Q X(R))
$$

and

$$
T_{Q, P}=T_{Q}^{-1} \circ T_{P}, \quad T_{P, Q}=T_{P}^{-1} \circ T_{Q}
$$

are both surjective. Actually (14) is a necessary and sufficient condition 
for $P X(R)$ and $Q X(R)$ to be canonically isomorphic. In particular, if $T_{P}$ and $T_{Q}$ are surjective, then $P X(R)$ and $Q X(R)$ are canonically isomorphic.

3. We are ready to prove that if $P \geq Q$, then the canonical injection $T_{Q, P}$ from $P X(R)$ into $Q X(R)$ exists $(X=B, D, E, B D, B E)$. Since $P \geq Q$ implies $u=P_{u}^{\Omega} \leq Q_{u}^{\Omega}$ for $u \in P X(R)^{+}, u \leq Q_{u}^{\Omega} \leq H_{u}^{\Omega} \leq T_{P} u$ and $\left\{Q_{u}^{\Omega}\right\}$ is increasing. Therefore

$$
T u=\lim _{\Omega \rightarrow R} Q_{u}^{\Omega}
$$

exists and $u-T u$ is a quasipotential in view of $|u-T u|=T u-u \leq$ $T_{P} u-u=\left|u-T_{P} u\right|$ for every $u \in P X(R)^{+}$. Since $P X(R)^{+}$generates $P X(R)$, this is also true for every $u \in P X(R)$ and $T$ is a linear operator from $P X(R)$ into $Q(R)$. We only have to show that $T u \in Q X(R)$ in order to conclude that $T=T_{Q, P}$. If $X=B$, then $\left|Q_{u}^{\Omega}\right| \leq\|u\|$ and $T u \in Q B(R)$. If $X=E$, then, by the energy principle and $Q \leq P$,

$$
E_{\Omega}^{Q}\left(Q_{u}^{\Omega}\right) \leq E_{\Omega}^{Q}(u) \leq E_{\Omega}^{P}(u) \leq E_{R}^{P}(u)
$$

Therefore, since $d Q_{u}^{\Omega} \wedge^{*} d Q_{u}^{\Omega} \rightarrow d T u \wedge^{*} d T u$, the Fatou lemma yields $E_{R}^{Q}(T u) \leq E_{R}^{P}(u)$ and $T u \in Q E(R)$. Finally let $u \in P D(R)$. We wish to show that $T u \in Q D(R)$. For this purpose we may assume that $u \in P D(R)^{+}$. Then, by $Q \leq P, 0 \leq u=P_{u}^{\Omega} \leq Q_{u}^{\Omega}$. Thus $E_{\Omega}^{Q}\left(Q_{u}^{\Omega}\right) \leq E_{\Omega}^{Q}(u)$ implies that

$$
\begin{aligned}
D_{\Omega}\left(Q_{u}^{\Omega}\right) & \leq D_{\Omega}(u)-\int_{\Omega}\left(Q_{u}^{\Omega}(z)\right)^{2} Q(z) d x d y+\int_{\Omega} u^{2}(z) Q(z) d x d y \\
& \leq D_{\Omega}(u)-\int_{\Omega} u^{2}(z) Q(z) d x d y+\int_{\Omega} u^{2}(z) Q(z) d x d y=D_{\Omega}(u) .
\end{aligned}
$$

Hence $D_{\Omega}\left(Q_{u}^{\Omega}\right) \leq D_{\Omega}(u) \leq D_{R}(u)$ and the Fatou lemma yields $D_{R}(T u) \leq$ $D_{R}(u)$, i.e. $T u \in Q D(R)$. Next we prove that $P X(R)$ and $(c P) X(R)$ are canonically isomorphic for every $c>0$. We only have to show this for $c \leq 1$. For, if $c>1$, then $(c P) X(R)$ is canonically isomorphic to $\left(c^{-1}(c P)\right) X(R)=P X(R)$ since $c^{-1}<1$. Then, since $P \geq c P$, the canonical injection $T_{c P, P}$ exists and by (13),

$$
T_{P}(P X(R)) \subset T_{c P}((c P) X(R)) \quad(\subset H X(R)),
$$

and by (14) we only have to show that this inclusion is improper, i.e. any $h \in T_{c P}((c P) X(R))$ belongs to $T_{P}(P X(R))$. Since $T_{c P}$ is order preserving and $(c P) X(R)^{+}$generates $(c P) X(R)$, we may assume that $h=T_{c P} v \geq 0$ with $v \in(c P) X(R)^{+}$. For brevity set $Q=c P$. Observe that $0 \leq P_{v}^{\Omega} \leq$ $Q_{v}^{\Omega}=v, P_{v}^{\Omega}=P_{h}^{\Omega}+P_{v-T_{Q}}^{\Omega}$, and $\left\{P_{v}^{\Omega}\right\}$ is decreasing. Thus 


$$
0 \leq u=\lim _{\Omega \rightarrow R} P_{v}^{\Omega}=\lim _{\Omega \rightarrow R} P_{h}^{\Omega} \leq v
$$

exists and $u \in P(R)^{+}$. By (8)

$$
h=v+\frac{1}{2 \pi} \int_{R} G_{R}(\cdot, \zeta) v(\zeta) c P(\zeta) d \xi d \eta
$$

and in particular

$$
\int_{R} G_{R}(\cdot, \zeta) v(\zeta) P(\zeta) d \xi d \eta<\infty
$$

Therefore, since $G_{\Omega}(\cdot, \zeta) P_{v}^{\Omega}(\zeta) \leq G_{R}(\cdot, \zeta) v(\zeta)$ and $h=\lim _{\Omega \rightarrow R} H_{v}^{\Omega}$, by applying the Lebesgue dominated convergence theorem to

$$
H_{v}^{\Omega}=P_{v}^{\Omega}+\frac{1}{2 \pi} \int_{R} G_{\Omega}(\cdot, \zeta) P_{v}^{\Omega}(\zeta) P(\zeta) d \xi d \eta
$$

as $\Omega \rightarrow R$, we obtain

$$
h=u+\frac{1}{2 \pi} \int_{R} G_{R}(\cdot, \zeta) u(\zeta) P(\zeta) d \xi d \eta .
$$

If $X=B$, then $\left|P_{v}^{a}\right| \leq\|v\|$ implies $\|u\|<\infty$ and a fortiori $u \in P B(R)^{+}$. Since $h-u$ is a potential, we must have $T_{P} u=h$. If $X=E$, then the energy principle implies that

$$
E_{\Omega}^{P}\left(P_{v}^{\Omega}\right) \leq E_{\Omega}^{P}\left((c P)_{v}^{\Omega}\right)=E_{\Omega}^{P}(v) \leq c^{-1} E_{\Omega}^{c P}(v) \leq c^{-1} E_{R}^{c P}(v) .
$$

The Fatou lemma yields $E_{R}^{P}(u)<\infty$ and $u \in P E(R)^{+}$. Similarly as above $h=T_{P} u$. Finally let $X=D$. By (9) and (10)

$$
D(v)=D(h)+\frac{1}{2 \pi} \int_{R \times R} G_{R}(z, \zeta) v(z) v(\zeta) c P(z) d x d y c P(\zeta) d \xi d \eta
$$

and we have $\langle v, v\rangle_{R}^{c P}<\infty$. Thus $\langle u, u\rangle_{R}^{P}=c^{-2}\langle u, u\rangle_{R}^{c P} \leq c^{-2}\langle v, v\rangle_{R}^{c P}<\infty$. Again by (9), $D(u)=D(h)+(1 / 2 \pi)\langle u, u\rangle_{R}^{P}<\infty$. Therefore $u \in P D(R)$ and as before $h=T_{P} u$. Combining two main assertions in this no., we maintain :

Proposition. If there exists a constant $c \geq 1$ such that $c^{-1} P \leq Q \leq c P$ on $R$, then $P X(R)$ and $Q X(R)$ are canonically isomorphic for $X=B, D, E, B D$, and $B E$.

Proof. Since $Q \geq c^{-1} P, T_{c^{-1} P, Q}$ exists. In view of that $\left(c^{-1} P\right) X(R)$ and $P X(R)$ are canonically isomorphic, $T_{P, c-1 P}$ exists and therefore by (6), 

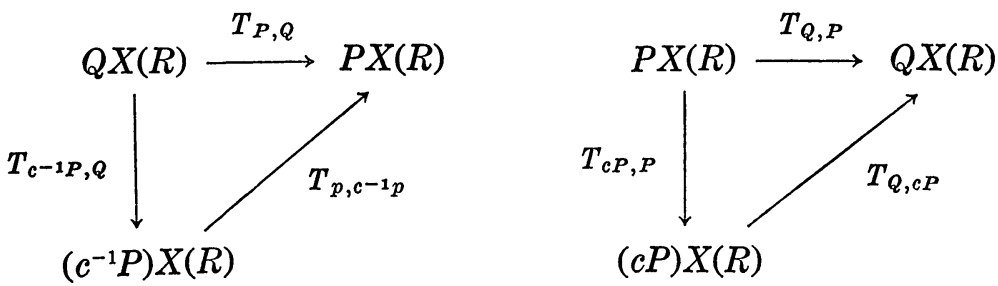

$T_{P, Q}=T_{P, c^{-1} P} \circ T_{c^{-1 P}, Q}$ exists. Similarly $T_{Q, P}$ exists (see the above diagrams) and therefore $P X(R)$ and $Q X(R)$ are canonically isomorphic.

Q.E.D.

\section{Canonical Extensions}

4. For convenience we say that an open subset $W$ of $R$ is normal if each point $z$ of the relative boundary $\partial W$ of $W$ posesses a parametric disk $U$ at $z$ such that $U \cap \partial W$ is a diameter of $U$. Hereafter we always use $W$ for normal open subsets of $R$. Consider the linear spaces

$$
P(W ; \partial W)=\{u \in P(W) \cap C(R) ; u \mid R-W=0\}
$$

and

$$
P X(W ; \partial W)=\{u \in P X(W) \cap C(R) ; u \mid R-W=0\}
$$

for $X=B, D, E, B D$, and $B E$. A linear operator $\lambda=\lambda_{P}=\lambda_{P}^{W}$ from $P X(W ; \partial W)$ into $P X(R)$ is said to be a canonical extension if $u-\lambda u$ is a quasipotential on $R$ for every $u \in P X(W ; \partial W)$. We write $H(W ; \partial W)$, $H X(W ; \partial W)$, and $\lambda_{H}$ for $P(W ; \partial W), P X(W ; \partial W)$, and $\lambda_{P}$ with $P \equiv 0$. As $P X(R), P X(W ; \partial W)$ is generated by $P X(W ; \partial W)^{+}$(cf. e.g. [9]). Similarly as in nos. 1 and 2 , we see that the canonical extension is unique, injective, order preserving, and isometric. We next prove that the canonical extension $\lambda_{P}$ always exists for $X=B, D, E, B D$, and $B E$ and for any $P$. First let $u \in P X(W ; \partial W)^{+}$. Since $u$ is subharmonic on $R, u \leq P_{u}^{\Omega} \leq P_{u}^{\Omega^{\prime}} \leq H_{u}^{\Omega^{\prime}}$ for $\Omega \subset \Omega^{\prime}$. If $X=B$, then $0 \leq P_{u}^{\Omega} \leq\|u\|$. If $X=E$, then $E_{\Omega}^{P}\left(P_{u}^{\Omega}\right) \leq$ $E_{\Omega}^{P}(u) \leq E_{R}^{P}(u)$. If $X=D$, then $E_{\Omega}^{P}\left(P_{u}^{\Omega}\right) \leq E_{\Omega}^{P}(u)$ implies that

$$
D_{\Omega}\left(P_{u}^{\Omega}\right) \leq D_{\Omega}(u)-\int_{\Omega}\left(\left(P_{u}^{\Omega}(z)\right)^{2}-u^{2}(z)\right) P(z) d x d y
$$

Since $\left(P_{u}^{\Omega}(z)\right)^{2}-u^{2}(z) \geq 0, \quad D_{\Omega}\left(P_{u}^{\Omega}\right) \leq D_{\Omega}(u) \leq D_{R}(u)$. In the latter two cases, the harmonic decomposition of $u$ yields the convergence of $\left\{H_{u}^{\Omega}\right\}$. Therefore

$$
\lambda_{P} u=\lim _{\Omega \rightarrow R} P_{u}^{\Omega}
$$


exists and belongs to $P X(R)$ for every $u \in P X(W ; \partial W)^{+}$and hence for every $u \in P X(W ; \partial W)$. Thus $\lambda=\lambda_{P}$ is a linear operator from $P X(W ; \partial W)$ into $P X(R)(X=B, D, E, B D, B E)$. Again let $u \in P X(W ; \partial W)^{+}$and $h \in H(R)$ with $u \leq h$. Then $u \leq P_{u}^{\Omega} \leq H_{u}^{\Omega} \leq H_{h}^{\Omega}=h$ implies that $\lambda_{H} u=\lim _{\Omega \rightarrow R} H_{u}^{\Omega} \leq h$ and thus $\lambda_{H} u$ is the least harmonic majorant of $u$. Therefore $\lambda_{H} u-u$ is a potential. Since $\left|u-\lambda_{P} u\right|=\lambda_{P} u-u \leq \lambda_{H} u-u, u-\lambda_{P} u$ is a quasipotential. For general $u \in P X(W ; \partial W)$, let $u=u_{1}-u_{2}$ with $u_{i} \in P X(W ; \partial W)^{+}$ $(i=1,2)$. Then $\left|u-\lambda_{P} u\right| \leq\left|u_{1}-\lambda_{P} u_{1}\right|+\left|u_{2}-\lambda_{P} u_{2}\right|$ shows that $u-\lambda_{P} u$ is a quasipotential, i.e. $\lambda_{P}$ is the canonical extension.

5. We denote by $P^{\prime}(R)\left(P^{\prime}(W ; \partial W)\right.$, resp.) the subspace of $P(R)$ $\left(P(W ; \partial W)\right.$, resp.) generated by $P(R)^{+}\left(P(W ; \partial W)^{+}\right.$, resp. $)$. Let $\chi=\chi_{W}$ be the characteristic function of $W$, i.e. $\chi \mid W=1$ and $\chi \mid R-W=0$. We define an operator $\mu=\mu_{P}=\mu_{P}^{W}$, which will be referred to as the canonical restriction, by

$$
\mu_{P} v=\lim _{\Omega \rightarrow R} P_{\chi v}^{W \cap \Omega}
$$

on $W$ and $\mu_{P} v=0$ on $R-W$. We use $\mu_{H}$ for $\mu_{P}$ with $P \equiv 0$. This is a linear operator from $P^{\prime}(R)$ into $P^{\prime}(W ; \partial W)$. To see that $\mu_{P}$ is well defined, let $v=v_{1}-v_{2}$ with $v_{i} \in P(R)^{+}(i=1,2)$. Since $0 \leq P_{x v_{i}}^{X \Omega_{0}} \leq v_{i}$ and $P_{x v_{i}}^{W \cap \Omega} \mid \partial W=0,\left\{P_{\chi v_{i}}^{W \cap \Omega}\right\}$ forms a decreasing net and thus $\lim _{\Omega \rightarrow R} P_{x v_{i}}^{W \cap \Omega}$ exists and belongs to $P(W ; \partial W)^{+}$if it is extended to $R$ by setting zero on $R-W$. From $P_{x v}^{W \cap \Omega}=P_{x v_{1}}^{W \cap \Omega}-P_{x v_{2}}^{W \cap \Omega}$, the existence of (17) in $P^{\prime}(W ; \partial W)$ follows. Observe that $P X(R) \subset P^{\prime}(R)$ and $P X(W ; \partial W) \subset P^{\prime}(W ; \partial W)$. The merit of considering $\mu_{P}$ lies in the following relation:

$$
\left(\mu_{P} \circ \lambda_{P}\right) u=u
$$

for every $u \in P X(W ; \partial W)(X=B, D, E, B D, B E)$. To prove this, it is sufficient to assume that $u \in P X(W ; \partial W)^{+}$. Since $P_{u}^{W \cap \Omega}=P_{x u}^{W \cap \Omega}=u$,

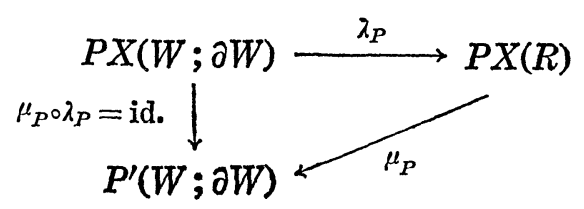

$P_{\chi \lambda_{P} u}^{W \cap \Omega}=u+P_{\chi\left(\lambda_{P} u-u\right)}^{W \cap \Omega}$. On the other hand, $0 \leq P_{\chi\left(\lambda_{P} u-u\right)}^{W \cap \Omega} \leq P_{\lambda_{P} u-u}^{\Omega} \rightarrow 0$ as $\Omega \rightarrow R$. Therefore $\mu_{P}\left(\lambda_{P} u\right)=u$. The relation (18) neither means that $\mu_{P}(P X(R)) \subset P X(W ; \partial W)$ nor that $\mu_{P}$ is injective on $P X(R)$ unless $\lambda_{P}$ is surjective. It only means that $\mu_{P}\left(\lambda_{P}(P X(W ; \partial W))=P X(W ; \partial W)\right.$ and $\mu_{P}$ 
is injective on $\lambda_{P}(P X(W ; \partial W))$. In this sense it is interesting to determine $W$ such that $\lambda_{P}^{W}: P X(W ; \partial W) \rightarrow P X(R)$ is surjective. Except for the case $X=B$ the problem seems to be very difficult (cf. [13]). Here we only give a sufficient condition.

6. In this no, we always assume that $R$ is connected. To discuss the surjectiveness of $\lambda_{P}$, we introduce three kinds of negligible sets. A subset $K$ of $R$ is said to be $X$-negligible ( $X=B, D$, and $B D$, resp.) if either $R$ is parabolic or there exists a normal open subset $W$ such that $K \subset R-W$ and $\lambda_{H}: H X(W ; \partial W) \rightarrow H X(R)(X=B, D$, and $B D$, resp. $)$ is surjective. We shall try to restate the concept in a more intuitively understandable term. First

$A$ subset $K$ of hyperbolic $R$ is $B$-negligible if and only if there exists a potential $p$ on $R$ such that $p \geq 1$ on $K$.

If $\lambda_{H}: H B(W ; \partial W) \rightarrow H B(R)$ is surjective with $K \subset R-W$, then $h=\mu_{H} 1 \in H B(W ; \partial W) 0 \leq h \leq 1$, and $\lambda_{H} h=1$. Since $h$ is subharmonic, $p=1-h$ is superharmonic. On the other hand $p=1-h=\left|h-\lambda_{H} h\right|$ is a quasipotential and thus $p$ is a potential. Clearly $p=1 \geq 1$ on $K$. Conversely assume the existence of such a $p$. By multiplying $p$ by a suitable constant and by choosing $W$ suitably, we can assume that $p \geq 1$ on $R-W$. Let $h \in H B(R)^{+}$. Then clearly $\mu_{H} h \in H B(W ; \partial W)$. Observe that $0 \leq h-\chi_{W} h \leq\|h\| p$ implies $0 \leq H_{h}^{W \cap \Omega}-H_{x W h}^{W \cap \Omega} \leq\|h\| p$. On letting $\Omega \rightarrow R, 0 \leq h-\mu_{H} h \leq\|h\| p$ on $W$ and trivially on $R-W$, and a fortiori $h-\mu_{H} h$ is a quasipotential on $R$. By $\left|h-\lambda_{H} \circ \mu_{H} h\right| \leq\left|\mu_{H} h-\lambda_{H}\left(\mu_{H} h\right)\right|$ $+\left|h-\mu_{H} h\right|$ we see that the subharmonic function $\left|h-\lambda_{H} \circ \mu_{H} h\right|$ is a quasipotential and thus $\lambda_{H} \circ \mu_{H} h=h$. Since $H B(R)^{+}$generates $H B(R)$, we conclude that $\lambda_{H}: H B(W ; \partial W) \rightarrow H B(R)$ is surjective. If we use the term of the compactification theory (cf. e.g. [1], [15]) we can restate the above assertion as follows: $\mathrm{K}$ is $B$-negligible if and only if the closure of $R-K$ in the Wiener compactification of $R$ is a neighborhood of the Wiener harmonic boundary. Next we prove

$A$ subset $K$ of hyperbolic $R$ is BD-negligible if and only if there exists a Dirichlet finite potential $p$ on $R$ such that $p \geq 1$ on $K$.

If $\lambda_{H}: H B D(W ; \partial W) \rightarrow H B D(R)$ is surjective, then $h=\mu_{H} 1$ belongs to $H B D(W ; \partial W), \quad 0 \leq h \leq 1$, and $\lambda_{H} h=1$. As above, $p=1-h$ is a potential, $p=1 \geq 1$ on $K$, and $D(p)=D(h)<\infty$. Conversely assume the existence of such a $p$ on $R$. On replacing $p$ by $p \cap 1=\min (p, 1)$, we may assume that $0 \leq p \leq 1$ on $R$ and $p=1$ on $K$. Let $h \in H B D(R)^{+}$. 
By the above, $\mu_{H} h \in H B(W ; \partial W)$ and $\lambda_{H}\left(\mu_{H} h\right)=h$. Since $H B D(R)^{+}$generates $H B D(R)$, we only have to show that $D_{W}\left(\mu_{H} h\right)<\infty$. Since $D_{R}((1-p) h)$ $<\infty$, by the harmonic decomposition of $(1-p) h$ (cf. e.g. [1], [15]) we see the existence of $k=\lim _{\Omega \rightarrow R} H_{(1-p) h}^{W \cap \Omega}$ in $H B D(W ; \partial W)$. Observe that $0 \leq h-(1-p) h \leq\|h\| p$ implies $0 \leq H_{h}^{W \cap \Omega}-H_{(1-p) h}^{W \cap \Omega} \leq\|h\| p$ and a fortiori $0 \leq h-k \leq\|h\| p$. Thus $\left|k-\mu_{H} h\right| \leq\left|\mu_{H} h-\lambda_{H}\left(\mu_{H} h\right)\right|+\|h\| p$, i.e. $\left|k-\mu_{H} h\right|$ is a quasipotential. Since $\left|k-\mu_{H} h\right|$ is subharmonic, we conclude that $\mu_{H} h=k \in H B D(W ; \partial W)$. Note that

$$
D\left(\mu_{H} h\right) \leq D(p h) .
$$

In terms of compactifications (cf. e.g. [1], [15]), we see that $K$ is $B D$ negligible if and only if the closure of $R-K$ in the Royden compactification of $R$ is a neighborhood of the Royden harmonic boundary. From these two characterizations and the first two trivial inclusions it follows that

$\{$ compact sets $\} \subset\{D$-negligible sets $\}$

$\subset\{B D$-negligible sets $\} \subset\{B$-negligible sets $\}$.

Although we do not give explicit examples here, it is not hard to see that the above inclusions are all strict. We are not successful in potential term characterization of $D$-negligible sets and only give the following sufficient condition:

$A$ subset $K$ of hyperbolic $R$ is $D$-negligible if there exists a Dirichlet finite potential $p$ on $R$ harmonic outside a compact set such that $p \geq 1$ on $K$.

The set $K_{\varepsilon, \zeta}=\left\{z \in R ; G_{R}(z, \zeta) \geq \varepsilon>0\right\}$ for any $\varepsilon$ is an example of a $D$-negligible set since $p=\left(G_{R}(\cdot, \zeta) \cap \varepsilon^{\prime}\right) / \varepsilon$ for large $\varepsilon^{\prime}$ is a potential as stated above. To prove the assertion suppose that $p \in H\left(R-\Omega_{0}\right)$. Since $p$ is a potential $\sup _{R-\Omega_{0}} p=\sup _{\partial \Omega_{0}} p=c<\infty$. On replacing $p$ by $p \cap c$, we may assume that $p$ is bounded. On applying the regularization we can also assume that $p \in C^{2}(R) \cap H\left(R-\Omega_{0}\right)$. Let $h \in H D(R)^{+}$and $h_{n}=h \wedge n$ $=$ the greatest harmonic minorant of $h$ and $n$. Then $D\left(h_{n}\right) \leq D(h), h_{n} \uparrow h$, and $D\left(h_{n}-h\right) \rightarrow 0$ (cf. e.g. [15]). Let $p_{\Omega}=p-H_{p}^{\Omega}$ for $\Omega \supset \Omega_{0}$ and let $k \in H D(R)$. By the Green formula

$$
\begin{aligned}
D_{\Omega}\left(p_{\Omega} k\right) & =-\int_{\Omega} p_{\Omega} k d^{*} d\left(p_{\Omega} k\right) \\
& =-\int_{\Omega_{0}} p_{\Omega} k^{2} d^{*} d p_{\Omega}-\frac{1}{2} \int_{\Omega} d p_{\Omega}^{2} \wedge^{*} d k^{2} \\
& =-\int_{\Omega} p_{\Omega} k^{2} d^{*} d p+\int_{\Omega} p_{\Omega}^{2} d k \wedge^{*} d k .
\end{aligned}
$$


By the Fatou lemma we conclude as $\Omega \rightarrow R$ that

$$
D(p k) \leq\|k\|_{\Omega_{0}}^{2} D(p)+\|p\|^{2} D(k) .
$$

By the second characterization above, $\mu_{H} h_{n} \in H D(W ; \partial W)$. By (19) and (20) we have

$$
D\left(\mu_{H} h_{n}-\mu_{H} h_{n+p}\right) \leq D(p)\left\|h_{n}-h_{n+p}\right\|_{\Omega_{0}}^{2}+\|p\|^{2} D\left(h_{n}-h_{n+p}\right) .
$$

Therefore $u=\lim _{n \rightarrow \infty} \mu_{H} h_{n} \in H D(W ; \partial W)$ and $D\left(u-\mu_{H} h_{n}\right) \rightarrow 0$ as $n \rightarrow \infty$. On the other hand, $D\left(\lambda_{H} u-h_{n}\right)=D\left(\lambda_{I I} u-\lambda_{H} \mu_{H} h_{n}\right) \leq D\left(u-\mu_{H} h_{n}\right)$ shows that $D\left(\lambda_{H} u-h\right)=0$, i.e. $\lambda_{H} u+a=h$ with a constant $a$. Take $e \in H B D(W ; \partial W)$ with $\lambda_{H} e=a$. Then $\lambda_{H}(u+e)=h$. Since $H D(R)^{+}$generates $H D(R)$, we conclude that $\lambda_{H}: H D(W ; \partial W) \rightarrow H D(R)$ is surjective.

7. In the definition of negligible sets we presupposed that $R$ is connected. The connectedness is not an essential restriction because we only have to consider componentwise if $R$ is not connected. If $R$ is parabolic, then $R$ itself is of degenerate character but does not quite match our definition in terms of the surjectiveness of $\lambda_{H}$. In this case $H X(R)=$ \{constants $\}$ and $H X(W ; \partial W)=\{0\}$ for $X=B, D, B D$ if $R-W \neq \phi$ (cf. e.g. [15]). From (8) in no. 2, it follows that $P X(R)=\{0\}$ and $P X(W ; \partial W)$ $=\{0\}$ for $X=B, D, E, B D, B E$ if $R$ is parabolic and $P \neq \equiv 0$. This unpleasant situation can be conventionally resolved if we includes nonnegative constants into the class of potentials when $R$ is parabolic. However, instead of providing such an artificial convention, we would rather avoid parabolic surfaces. The role of negligible sets is clarified by the

Proposition. Let $W$ be a normal open set in a hyperbolic connected Riemann surface $R$. If $R-W$ is $B$-negligible, then $\lambda_{P}: P B(W ; \partial W) \rightarrow P B(R)$ is surjective for every $P$; if $R-W$ is $D$-negligible, then $\lambda_{P}: P X(W ; \partial W)$ $\rightarrow P X(R)(X=B D, B E)$ is surjective for every $P$; if $R-W$ is $D$-negligible, then $\lambda_{P}: P X(W ; \partial W) \rightarrow P X(R)(X=D, E)$ is surjective for every $P$.

Proof. Suppose that $R-W$ is $B$-negligible. Take a potential $p$ on $R$ such that $p \geq 1$ on $R-W$. Let $u \in P B(R)^{+}$. Clearly $\mu_{P} u \in P B(W ; \partial W)$. Observe that $0 \leq u-\chi_{W} u \leq\|u\| p$ on $R$. Thus $0 \leq P_{u}^{W \cap \Omega}-P_{x_{W} u}^{W \cap \Omega} \leq\|u\| p$ and a fortiori $0 \leq u-\mu_{P} u \leq\|u\| p$, i.e. $u-\mu_{P} u$ is a quasipotential. By $\left|u-\lambda_{P} \circ \mu_{P} u\right| \leq\left|\mu_{P} u-\lambda_{P}\left(\mu_{P} u\right)\right|+\left|u-\mu_{P} u\right|$, we see that the subharmonic function $\left|u-\lambda_{P} \circ \mu_{P} u\right|$ is a quasipotential and therefore $\lambda_{P} \circ \mu_{P} u=u$. Since $P B(R)^{+}$generates $P B(R), \lambda_{P}: P B(W ; \partial W) \rightarrow P B(R)$ is surjective. 
Next suppose $R-W$ is $B D$-negligible. As in no. 6, take a Dirichlet finite potential $p$ such that $0 \leq p \leq 1$ and $p=1$ on $R-W$. Let $u \in P B Y(R)^{+}$ $(Y=D, E)$. Since $R-W$ is $B$-negligible, $\mu_{P} u \in P B(W ; \partial W)$ and $\lambda_{P} \mu_{P} u=u$. Since $P B Y(R)^{+}$generates $P B Y(R)$, we can conclude the surjectiveness of $\lambda_{P}: P B Y(W ; \partial W) \rightarrow P B Y(R)$ if we show $Y\left(\mu_{P} u\right)<\infty(Y=D, E)$. First let $u \in P B D(R)^{+}$, i.e. $D(u)<\infty$. Observe that

$$
u=T_{P} u-\frac{1}{2 \pi} \int_{R} G_{R}(\cdot, \zeta) u(\zeta) P(\zeta) d \xi d \eta,
$$

$P_{\chi_{W} u}^{W \cap \Omega} \downarrow \mu_{P} u, \quad G_{W \cap \Omega}(\cdot, \zeta) P_{\chi_{W}}^{W \cap \Omega} \leq G_{R}(\cdot, \zeta) u(\zeta)$, and $H_{\chi_{W}}^{W \cap \Omega} \rightarrow \mu_{H} T_{P} u$ since $\chi_{W} u$ $-\chi_{W} T_{P} u$ is a quasipotential. By the Lebesgue dominated convergence theorem,

$$
P_{\chi_{W} u}^{W \cap \Omega}=H_{\chi_{W}}^{W \cap \Omega}-\frac{1}{2 \pi} \int_{W \cup \Omega} G_{W \cap \Omega}(\cdot, \zeta) P_{\chi_{W}}^{W \cap \Omega}(\zeta) P(\zeta) d \xi d \eta
$$

implies that

$$
\mu_{P} u=\mu_{H} T_{P} u-\frac{1}{2 \pi} \int_{W} G_{W}(\cdot, \zeta) \mu_{P} u(\zeta) P(\zeta) d \xi d \eta
$$

Since $D(u)=D\left(T_{P} u\right)+(1 / 2 \pi)\langle u, u\rangle_{R}^{P}<\infty, T_{P} u \in H B D(R)$, and a fortiori the surjectiveness of $\lambda_{H}$ implies that $D\left(\mu_{H} T_{P} u\right)<\infty$. Therefore, by $0 \leq \mu_{P} u \leq u$, we see that

$$
\begin{aligned}
D\left(\mu_{P} u\right)= & D\left(\mu_{H} T_{P} u\right)+\frac{1}{2 \pi}\left\langle\mu_{P} u, \mu_{P} u\right\rangle_{W}^{P} \\
& \leq D\left(\mu_{H} T_{P} u\right)+\frac{1}{2 \pi}\langle u, u\rangle_{R}^{P}<\infty .
\end{aligned}
$$

Next let $E^{P}(u)<\infty$. Since $D(u)<\infty$ by the above we have $D\left(\mu_{P} u\right)<\infty$. Therefore $0 \leq \mu_{P} u \leq u$ implies that

$$
\begin{aligned}
E^{P}\left(\mu_{P} u\right)= & D\left(\mu_{P} u\right)+\int_{R}\left(\mu_{P} u(\zeta)\right)^{2} P(\zeta) d \xi d \eta \\
& \leq D\left(\mu_{P} u\right)+\int_{R} u^{2}(\zeta) P(\zeta) d \xi d \eta \\
& \leq D\left(\mu_{P} u\right)+E^{P}(u)<\infty
\end{aligned}
$$

Finally suppose that $R-W$ is $D$-negligible. Since $P X(R)^{+}$generates $P X(R)$, we only have to show that $\mu_{P} u \in P X(W ; \partial W)$ and $\lambda_{P} \mu_{P} u=u$ for every $u \in P X(R)^{+}(X=D, E)$. By exactly the same proof as above we 
see that $\mu_{P} u \in P X(W ; \partial W)$. In the above proof, $D\left(\mu_{H} T_{P} u\right)<\infty$ followed from the surjectiveness of $\lambda_{H}: H B D(W ; \partial W) \rightarrow H B D(R)$. In the present case it follows from that of $\lambda_{H}: H D(W ; \partial W) \rightarrow H D(R)$. Thus we only have to show that $\lambda_{P} \mu_{P} u=u$. Set $\mu_{P} u=\mu_{H} T_{P} u-p$, where $p=(1 / 2 \pi)$ $\cdot \int G_{W}(\cdot, \zeta) \mu_{P} u(\zeta) P(\zeta) d \xi d \eta$ is a quasipotential since it is dominated by $(1 / 2 \pi) \int G_{R}(\cdot, \zeta) u(\zeta) P(\zeta) d \xi d \eta$, a potential. Observe that

$$
\begin{aligned}
P_{\mu_{P} u}^{\Omega} & =H_{\mu_{P} u}^{\Omega}-\frac{1}{2 \pi} \int_{\Omega} G_{\Omega}(\cdot, \zeta) P_{\mu_{P} u}^{\Omega}(\zeta) P(\zeta) d \xi d \eta \\
& =H_{\mu_{H} T_{P} u}^{\Omega}-H_{p}^{\Omega}-\frac{1}{2 \pi} \int_{\Omega} G_{\Omega}(\cdot, \zeta) P_{\mu_{P} u}^{\Omega}(\zeta) P(\zeta) d \xi d \eta .
\end{aligned}
$$

Since $0 \leq P_{\mu_{P} u}^{\Omega} \leq u$ and $\lim _{\Omega \rightarrow R} H_{p}^{\Omega}=0$, the Lebesgue dominated convergence theorem yields

$$
\lambda_{P} \mu_{P} u=\lambda_{H} \mu_{H} T_{P} u-\frac{1}{2 \pi} \int_{R} G_{R}(\cdot, \zeta) \lambda_{P} \mu_{P} u(\zeta) P(\zeta) d \xi d \eta .
$$

i.e. $T_{P} \lambda_{P} \mu_{P} u=\lambda_{H} \mu_{H} T_{P} u$. Since $\lambda_{H}: H D(W ; \partial W) \rightarrow H D(R)$ is surjective, $\lambda_{H} \mu_{H} T_{P} u=T_{P} u$ and thus $T_{P} \lambda_{P} \mu_{P} u=T_{P} u$. The injectiveness of $T_{P}$ implies that $\lambda_{P} \mu_{P} u=u$.

Q.E.D.

8. We now complete the proof of our order comparison theorem stated in the introduction. If $R$ is parabolic, then $H X(R)=$ constants $(X=B, D, B D$ ) (cf. e.g. [15]) and by (8) in no. $2 P X(R)=\{0\}$ for $P \neq \equiv$ and $X=B, D, E, B D$ and $B E$. Thus the comparison question is of interest only for the case $R$ is hyperbolic. Suppose (1) is valid on $R$ except for a $B$-negligible set $K$. Let $W$ be a normal open subset of $R$ such that $R-W \supset K$ and $R-W$ is $B$-negligible. Since (1) is valid on the whole $W$, a Riemann surface, Proposition 3 assures that there exists the canonical isomorphism $T_{Q, P}^{W}$ of $P B(W)$ onto $Q B(W)$. By using (12), it is not hard to see that $T_{Q, P}^{W}$ may be considered as a linear isomorphism of $P B(W ; \partial W)$ onto $Q B(W ; \partial W)$. By Proposition $7, \lambda_{Q} \circ T_{Q, P}^{W} \circ \mu_{P}$ is a linear isomorphism of $P B(R)$ onto $Q B(R)$. To see that $\lambda_{Q} \circ T_{Q, P}^{W} \circ \mu_{P}=T_{Q, P}$ is the canonical isomorphism of $P B(R)$ onto $Q B(R)$, we have

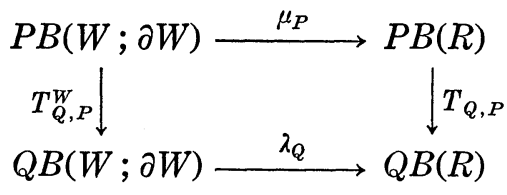


to show that $u-T_{Q, P}^{W} u$ is a quasipotential on $R$ for every $u \in P B(W ; \partial W)$. We may assume that $u \geq 0$. Since $u-T_{Q P}^{W} u$ is a quasipotential on $W$, $T_{P}^{W} u=T_{Q}^{W}\left(T_{Q, P}^{W} u\right)=h \in H B(W ; \partial W)$. Observe that

$$
u=h-\frac{1}{2 \pi} \int_{W} G_{W}(\cdot, \zeta) u(\zeta) P(\zeta) d \xi d \eta
$$

and similarly

$$
T_{Q, P}^{W} u=h-\frac{1}{2 \pi} \int_{W} G_{W}(\cdot, \zeta) T_{Q, P}^{W} u(\zeta) Q(\zeta) d \xi d \eta .
$$

Therefore by $u \leq \lambda_{P} u \equiv v$ and $T_{Q, P}^{W} u \leq \lambda_{Q} T_{Q, P}^{W} u \equiv w$,

$$
\begin{aligned}
\left|u-T_{Q, P}^{W} u\right| & \leq \frac{1}{2 \pi} \int_{W} G_{W}(\cdot, \zeta)(v(\zeta) P(\zeta)+w(\zeta) Q(\zeta)) d \xi d \eta \\
& \leq \frac{1}{2 \pi} \int_{R} G(\cdot, \zeta)(v(\zeta) P(\zeta)+w(\zeta) Q(\zeta)) d \xi d \eta \\
= & \left(T_{P} v-v\right)+\left(T_{Q} w-w\right)<\infty
\end{aligned}
$$

i.e. $\left|u-T_{Q, P}^{W} u\right|$ is dominated by the potential $\left(T_{P} v-v\right)+\left(T_{Q} w-w\right)$. By the similar applications of Propositions 3 and 7 as above, the other part of our comparison theorem can be proven verbatimly.

\section{Appendix: Integral Comparisons}

9. The order comparison (1) is very handy in many practical applications (cf. e.g. [8], [12]). However it is very far from being necessary. In pursuing the complete condition for the existence of canonical isomorphisms it is indespensable to consider the so-called integral comparisons. We denote by $G_{R}^{P}(z, \zeta)$ the Green's function of the equation $\Delta u=P u$ on $R$. Hence $G_{R}(z, \zeta)=G_{R}^{P}(z, \zeta)$ with $P \equiv 0$. Let $W$ be a normal open subset. We say that $(P, Q)$ satisfies the condition (B) on $W$ if

$$
\left\{\begin{array}{l}
\int_{W} G_{W}^{P}(\cdot, \zeta)|Q(\zeta)-P(\zeta)| d \xi d \eta<\infty \\
\int_{W} G_{W}^{Q}(\cdot, \zeta)|P(\zeta)-Q(\zeta)| d \xi d \eta<\infty
\end{array}\right.
$$

We say that $(P, Q)$ satisfies the condition (D) on $W$ if

$$
\left\{\begin{array}{l}
\int_{W \times W} G_{W}^{P}(z, \zeta)|Q(z)-P(z)| \cdot|Q(\zeta)-P(\zeta)| d x d y d \xi d \eta<\infty, \\
\int_{W \times W} G_{W}^{Q}(z, \zeta)|P(z)-Q(z)| \cdot|P(\zeta)-Q(\zeta)| d x d y d \xi d \eta<\infty .
\end{array}\right.
$$


Finally we say that $(P, Q)$ satisfies the condition (E) on $W$ if

$$
\int_{W}|P(\zeta)-Q(\zeta)| d \xi d \eta<\infty
$$

In our former paper [8] we showed that if $(P, Q)$ satisfies the condition $(X)$ on $R$, then $P B X(R)$ and $Q B X(R)$ are canonically isomorphic $(X=B, D, E, ; B B=B)$. If we use this in the proof in no. 8 instead of Proposition 3, then we obtain:

INTEgRal COMPaRison THEOREM 1. If $R-W$ is B-negligible and $(P, Q)$ satisfies the condition $(B)$ on $W$, then $P B(R)$ and $Q B(R)$ are canonically isomorphic; If $R-W$ is $B D$-negligible and $(P, Q)$ satisfies the condition (D) ((E), resp.), then $P B D(R)(P B E(R)$, resp.) and $Q B D(R)(Q B E(R)$, resp.) are canonically isomorphic.

The prototypes of this theorem are found in [6], Maeda [5], GlasnerKatz [2], etc. The integral conditions for $P X(R)$ and $Q X(R)(X=D, E)$ to be canonically isomorphic are not known. To find them is one of very important open problem on canonical isomorphisms. It may be instructive to point out that even if $P B X(R)$ and $Q B X(R)$ are canonically isomorphic, $P X(R)$ and $Q X(R)$ need not be canonically isomorphic for $X=D, E$ (cf. Singer [17], [11]), although $P B X(R)(X=D, E)$ are dense in $P X(R)$.

10. The above theorem applied to $(P, 0)$ takes the more precise form. First, since $\int_{R} G_{R}^{P}(\cdot, \zeta) P(\zeta) d \xi d \eta \leq 2 \pi$ and $G_{R}^{P}(\cdot, \zeta) \leq G_{R}(\cdot, \zeta)$, the condition

$$
\int_{W} G_{R}(\cdot, \zeta) P(\zeta) d \xi d \eta<\infty
$$

implies (B) for $(P, 0)$ on $W$, the condition

$$
\int_{W \times W} G_{R}(z, \zeta) P(z) P(\zeta) d x d y d \xi d \eta<\infty
$$

implies (D) for $(P, 0)$ on $W$, and condition

$$
\int_{W} P(\zeta) d \xi d \eta<\infty
$$

trivially implies (E) for $(P, 0)$ on $W$. Thus the condition $\left(\mathrm{B}_{0}\right)$ for $B$-negligible $R-W$ implies the surjectiveness of $T_{P}: P B(R) \rightarrow H B(R)$. 
The condition $\left(X_{0}\right)(X=D, E)$ for $B D$-negligible $R-W$ implies the surjectiveness of $T_{P}: P B X(R) \rightarrow H B X(R)$. Conversely assume $T_{P}: P X(R) \rightarrow$ $H X(R)$ is surjective and let $e=T_{P}^{-1} 1(X=B, B D, B E)$. We can choose a normal open set $W$ such that

$$
\left\{z \in R ; e(z) \geq \frac{1}{2}\right\} \subset W \subset\left\{z \in R ; e(z)>\frac{1}{3}\right\} .
$$

By using relations

$$
1=e+\frac{1}{2 \pi} \int_{R} G_{R}(\cdot, \zeta) e(\zeta) P(\zeta) d \xi d \eta
$$

and

$$
D(e)=\frac{1}{2 \pi} \int_{R \times R} G_{R}(z, \zeta) e(z) e(\zeta) P(z) d x d y P(\zeta) d \xi d \eta,
$$

we derive $\left(\mathrm{B}_{0}\right)\left(\left(\mathrm{D}_{0}\right)\right.$, resp.) on $W$ if $X=B(D$, resp.). If $X=E$, then

$$
E_{R}^{P}(e)=D(e)+\int_{R} e^{2}(\zeta) P(\zeta) d \xi d \eta<\infty
$$

implies $\left(\mathrm{E}_{0}\right)$ on $W$. Since $p=2(1-e)=\frac{1}{\pi} \int_{R} G_{R}(\cdot, \zeta) e(\zeta) P(\zeta) d \xi d \eta$ is a potential and $p \geq 1$ on $R-W, R-W$ is $B$-negligible. If $X=D$ or $E$, then $D(e)<\infty$ and a fortiori $D(p)<\infty$, i.e. $R-W$ is $B D$-negligible. Thus we have shown (cf. [6], Glasner-Nakai [3], Glasner-Katz [2])

INTEGRAL COMPARISON THEOREM 2. The linear spaces $P B(R)$ and $H B(R)$ are canonically isomorphic if and only if there exists a normal open subset $W$ on which $\left(\mathrm{B}_{0}\right)$ is valid such that $R-W$ is B-negligible; $P B D(R)(P B E(R)$, resp.) and $H B D(R)(H B D(R)$, resp.) are canonically isomorphic if and only if there exists a normal open subset $W$ on which $\left(\mathrm{D}_{0}\right)\left(\left(\mathrm{E}_{0}\right)\right.$, resp. $)$ is valid such that $R-W$ is BD-negligible.

Again the condition when $T_{P} ; P X(R) \rightarrow H X(R)(X=D, E)$ is surjective has not yet been obtained. Examples are known that $T_{P}: P B X(R)$ $\rightarrow H B X(R)$ is surjective but $T_{P}: P X(R) \rightarrow H X(R)$ is not for $X=D, E$ (Singer [17], [11]). To seek the (complete) condition for $T_{P}: P X(R) \rightarrow$ $H X(R)(X=D, E)$ to be surjective seems to be urgently important.

\section{REFERENCES}

[1] C. Constantinescu-A. Cornea: Ideale Ränder Riemannscher Flächen, Springer, 1963. 
[2] M. Glasner-R. Katz: On the behavior of solutions of $\Delta u=P u$ at the Royden boundary, J. d'Analyse Math., 22 (1969), 345-354.

[3] M. Glasner-M. Nakai: Riemannian manifolds with discontinuous metrics and the Dirichlet integral, Nagoya Math., J., 46 (1972), 1-48.

[4] P. Loeb: An axiomatic treatment of pairs of elliptic differential equations, Ann. Inst. Fourier, 16 (1966), 167-208.

[5] F.-Y. Maeda: Boundary value problems for the equation $\Delta u-q u=0$ with respect to an ideal boundary, J. Sci. Hiroshima Univ., 32 (1968), 85-146.

[6] M. Nakai: The space of bounded solutions of the equation $\Delta u=P u$ on a Riemann surface, Proc. Japan Acad., 36 (1960), 267-272.

[ 7 ] — : The space of Dirichlet-finite solutions of the equation $\Delta u=P u$ on a Riemann surface, Nagoya Math. J., 18 (1961), 111-131.

[ 8 ] - : The equation $\Delta u=P u$ on $E^{m}$ with almost rotation free $P \geqq 0$, Tôhoku Math. J., 23 (1971), 413-431.

[9] ——: Dirichlet finite solutions of $\Delta u=P u$ on open Riemann surfaces, Kôdai Math. Sem. Rep., 23 (1971), 385-397.

[10] - The equation $\Delta u=P u$ on the unit disk with almost rotation free $P \geq 0$, J. Diff. Eq., 11 (1972), 307-320.

[11] —-: Canonical isomorphisms of energy finite solutions of $\Delta u=P u$ on open Riemann surfaces, Proc. Japan Acad. (to appear).

[12] —-: Uniform densities on hyperbolic Riemann surfaces, Nagoya Math. J., 51 (1973) (to appear).

[13] - : Banach spaces of bounded solutions of $\Delta u=P u(P \geq 0)$ on hyperbolic Riemann surfaces (to appear).

[14] H. Royden: The equation $\Delta u=P u$, and the classification of open Riemann surfaces, Ann. Acad. Sci. Fenn., 271 (1959), 1-27.

[15] L. Sario-M. Nakai: Classification Theory of Riemann Surfaces, Springer, 1970.

[16] I. Singer: Dirichlet finite solutions of $\Delta u=P u$, Proc. Amer. Math. Soc., 32 (1972), 464-468.

[17] —-: Boundary isomorphism between Dirichlet finite solutions of $\Delta u=P u$ and harmonic functions, Nagoya Math. J., 50 (1973), 7-20.

* To contact the author: 52 Eguchi, Hinaga, Chita, Aichi, Japan.

** The work was supported by Grant DA-ARO-D-31-124-71-G20 (UCLA).

Nagoya University 